Volume 1 Issue 2

December 2016

\title{
International and unifying standards of pre-hospital care
}

Shane Knox PhD

Irish College of Paramedics

National Ambulance Service, Ireland.

\section{Recommended Citation}

Knox S. International and unifying standards of pre-hospital care. Irish Journal of Paramedicine. 2016 Dec; $1(2)$

This is an Open Access article distributed under the terms of the Creative Commons Attribution-Non-Commercial-ShareAlike 4.0 International (http://creativecommons.org/licenses/by-nc-sa/4.0/), which permits use, distribution, and reproduction in any medium, provided the original work and any attributes thereof are properly cited, are distributed under the same licence, and that the work is not used for commercial purposes.

Follow the Irish Journal of Paramedicine online at www.irishparamedicine.com, on Twitter (@irishjparamed) and on Facebook. 
EDITORIAL

\title{
International and Unifying Standards of Pre-hospital Care
}

\author{
Shane Knox $\mathrm{PhD}^{1}$
}

1. President, Irish College of Paramedics

Correspondence: Dr. Shane Knox, Irish College of Paramedics, 4 Keeper Road, Drimnagh, Dublin 12. Email: icopinfo@gmail.com

In Ireland, as with most other developed countries, the provision of pre-hospital care has improved significantly, particularly over the last two decades. The establishment of regulatory bodies (1-3) to provide governance and oversight for pre-hospital providers has been an important step in improving the level of pre-hospital care.

There are varying models of pre-hospital care ranging from the professional paramedic provision of care through the mix of first aid (4) Basic Life Support (BLS), Immediate care, Advanced Life Support (ALS) and the many associated specialist paramedicine grades. This model of paramedic care is typically associated with established systems of prehospital care in countries with recognised professional bodies, with or without the associated regulator including Canada, the USA, New Zealand, South Africa, Australia, the United Kingdom, and Ireland. Depending where you are in the world an ambulance is staffed by varying numbers and differing practitioner qualifications. Discussions on the benefits, or not, of the various types of paramedic /EMS practitioners continues to try to determine which combination of grade provides the most appropriate level of care, ALS (57) or BLS (8), or a mixed model of crewing?

Another model of care is the physician-led approach or the 'Franco-German' model of EMS. This model is also an effective model of care and is utilised in countries such as Austria and Malta, as well as Germany and France. Studies showing the advantages of having a physician-led prehospital response are interesting and show, for example, how the success rate of intubations improve if the difficult airway is managed by an emergency physician (9). Or how a transport helicopter with a physician on-board has an important role to play by allowing admissions to specialised centres (10). There are discussions on the type of specialist required and the required competencies for that physician practicing in the EMS field. Should the physician be an anaesthesiologist as commonly deployed in Nordic countries (11) or a consultant, or other senior grade, in Emergency Medicine? Is there a need for an increased role of the General Practitioner through a type of collaboration between the Emergency Department and General Practice for urgent care collaboration (12) or an increased requirement to move the GP from the practice-based model towards the large scale GP cooperative model?(13)

Another model of care is the nurse-led model described as the 'new profession in the pre-hospital care field' (14) in countries such as Sweden, or a mixed model where nurses work with paramedics (15) or in the Netherlands where an ambulance is staffed by a 'registered ambulance driver' and a 'registered nurse' (16). There are many studies comparing the various models of pre-hospital care from nurse versus physician (17), physician versus paramedic $(16,18)$ the benefits of having HEMS physicians (19) or the benefit in having HEMS nurses who demonstrate a higher rate of success with intubation compared to paramedics. (20)

Having considered the various alternatives in response type, and without trying to determine which one is best, we should decide firstly which model is required for our particular demographics and region, what are the common type of calls and what are the required competencies of those responding? Consideration should also be given to the proximity of the most appropriate facility, and not necessarily hospital, for the patient to be transferred to, if indeed they need transferred at all.

Internationally there are so many models of prehospital care but for example the efficient model of physician-led HEMS utilised in New South Wales, Australia linked to a major trauma centre (21) may not be the model for use in Ireland. Ireland currently has an aeromedical unit provided by the Irish Defence Forces which is clinically staffed by a National Ambulance Service Advanced Paramedic and a Defence Forces EMT. In addition Ireland also has access to four strategically situated Irish Coastguard search-and-rescue helicopters staffed by paramedics. Currently there are discussions regarding the introduction of physician-led HEMS in the south of the country. So in the small island of Ireland we will have six helicopters, from three organisations with three different models of deployment and staffing. This is great, but where's the evidence and what type of staffing is optimal, efficient, and effective?

Perhaps there are better ways to deploy physicians for pre-hospital care in the absence of developing extended skills for paramedics? For example, if paramedics are unable to replace urinary catheters for the patient with agonising pain at home or in the nursing home and the patient has to be taken out of the comfort of their residence to transfer them to an overcrowded ED, to wait and have the issue resolved, then this is an ideal task for the physician. The call could be appropriately triaged and passed to a physician to respond and perform the required procedure, thus removing the necessity of having to remove the patient at all. Many other examples of non-emergency low-acuity calls exist and physicians could be effectively utilised. Compare the evidence; let's see how many catheter changes are required compared to the many Rapid Sequence Inductions which are required, genuinely required, pre-hospital? 
There is a role for physicians in pre-hospital care, there is no doubt about that. However the call triage or paramedic decision to call for physician assistance would appear more appropriate than sending physicians to calls where skilled EMTs, paramedics and advanced paramedics can manage the patient within their current scope of practice. In Ireland, paramedics can also call for aeromedical support which allows crews to handover to an advanced paramedic for immediate evacuation and effective advanced care.

As well as the various models of care there is a growing need to develop an international standard of paramedic and all of the associated grades. There are many examples of standards of education and of paramedic competencies (22-26) and guidelines (27-29). The profession has made significant strides over the years but there are many different standards of practitioner with different skills and scope of practice. Perhaps soon, an EMT certified in one country will be automatically recognised internationally. Maybe when someone has a paramedic license from their national regulator it will mean something outside their own country and will be valued in other countries. Imagine one international set of pre-hospital practitioner standards which standardise the various roles, skills, and competencies and provide automatic recognition in every other country. Is this too much to imagine? Perhaps with agreements between national paramedic professional bodies or respective regulatory bodies, we can develop an international acceptable minimal standard for the many levels of pre-hospital care practitioner.

We might then decide the composition of the responding crew, the skills of the responders and practitioners and the inclusion of other professions all tasked to appropriate calls to match their skills and scope of practice with the ultimate focus on effective patient care.

Author contributions: SK was author of the manuscript, and performed the final editing.

Source of support/funding: None.

Conflict of interest: SK is President of the Irish College of Paramedics, and an Editorial Board member of the IJP.

Provenance and review: Commissioned, not peerreviewed.

\section{References}

1. Health and Care Professions Council (UK). (2016) Accessed 15th November 2016; Available from: http:// www.hpc-uk.org/.

2. Health Professions Council of South Africa. HPCSA Professional Boards. (2016) Accessed 16th November 2016; Available from: http://www.hpcsa.co.za/PBEmergencyCare.

3. Pre-Hospital Emergency Care Council (2016) Accessed 16th November 2016; Available from: https:/www.phecit.ie/. 4. Tanigawa, K. and K. Tanaka (2006) Emergency medical service systems in Japan: Past, present, and future. Resuscitation, 69(3): p. 365-370.
5. Eckstein, M., et al. (2000) Effect of Prehospital Advanced Life Support on Outcomes of Major Trauma Patients. Journal of Trauma and Acute Care Surgery, 48(4): p. 643-648.

6. Stiell , I.G., et al. (2004) Advanced Cardiac Life Support in Out-of-Hospital Cardiac Arrest. New England Journal of Medicine, 351(7): p. 647-656.

7. Jayaraman, S., D. Sethi, and R. Wong (2014) Advanced training in trauma life support for ambulance crews. The Cochrane Library.

8. Sanghavi, P., et al. (2015) Outcomes after out-of-hospital cardiac arrest treated by basic vs advanced life support. JAMA Internal Medicine, 175(2): p. 196-204.

9. Thoeni, N., et al. (2015) Incidence of difficult airway situations during prehospital airway management by emergency physicians-A retrospective analysis of 692 consecutive patients. Resuscitation, 90: p. 42-45.

10. Tazarourte, K., et al. (2013) Update on prehospital emergency care of severe trauma patients. Annales Françaises d'Anesthésie et de Réanimation, 32(7-8): p. 477-482.

11. Langhelle, A. et al. (2004) International EMS Systems: the Nordic countries. Resuscitation, 61(1): p. 9-21.

12. van Gils-van, E. et al. (2015) Out-of-Hours Care Collaboration between General Practitioners and Hospital Emergency Departments in the Netherlands. The Journal of the American Board of Family Medicine, 28(6): p. 807-815.

13. van Charante $M$ et al. (2007) Out-of-hours demand for GP care and emergency services: patients' choices and referrals by general practitioners and ambulance services. BMC Family Practice, 8(1): p. 46.

14. Suserud, B (2005) A new profession in the pre-hospital care field - the ambulance nurse. Nursing in Critical Care, 10 (6): p. 269-271.

15. Williams R (2012) Nurses who work in the ambulance service. Emergency Nurse, 20(2): p. 14-17.

16. deVries G, Luitse J (2001) Emergency medicine in the Netherlands. Annals of Emergency Medicine, 38(5): p. 583587.

17. van Schuppen H, Bierens J (2011) Understanding the prehospital physician controversy. Step 1: comparing competencies of ambulance nurses and prehospital physicians. European Journal of Emergency Medicine, 18(6): p. 322-327.

18. Roudsari, B et al. (2007) International comparison of prehospital trauma care systems. Injury, 38(9): p. 993-1000.

19. van Schuppen H Bierens J (2015) Understanding the prehospital physician controversy. Step 2: analysis of onscene treatment by ambulance nurses and helicopter emergency medical service physicians. European Journal of Emergency Medicine, 22(6): p. 384-390.

20. Peters J. et al. (2015) First-pass intubation success rate during rapid sequence induction of prehospital anaesthesia by physicians versus paramedics. European Journal of Emergency Medicine, 22(6): p. 391-394.

21. Taylor, C. et al. (2012) The cost-effectiveness of physician staffed Helicopter Emergency Medical Service 
(HEMS) transport to a major trauma centre in NSW, Australia. Injury, 43(11): p. 1843-1849.

22. Paramedic Association of Canada. National occupational competency profile for paramedics 2011. Accessed 25th November 2016. Available from: http:// www.paramedic.ca/site/nocp?nav $=02$.

23. Paramedics Australasia (2011) Australasian Competency Standards. Available from: http://www.paramedics.org/ content/2011/10/PA_Australasian-Competency-Standards-for -paramedics_July-20111.pdf.

24. Health and Care Professions Council UK (2014) Standards of Proficiency - Paramedics. Accessed 25th November 2016; Available from: http://www.hpc-uk.org/ assets/

documents/1000051CStandards_of_Proficiency_paramedics. pdf.

25. Pre-Hospital Emergency Care Council. (2011) PHECC Education and Training Standards. Accessed 25th November 2016; Available from: http://www.phecit.ie/PHECC/ Education_and_careers/Education_and_training_standards/ PHECC/Education_and_careers/

Education_and_Training_Standards/

Education_and_Training_Standards.aspx?Hkey=1733bd9d-

1ea7-41e7-af20-8ab0f40ab846.

26. Health Professions Council of South Africa (2016) Emergency Care Assistant Curriculum. Accessed 25th November 2016; Available from: http://www.hpcsa.co.za/ uploads/editor/UserFiles/

ECA_CURRICULUM_18_MARCH_2016.pdf.

27. Machingaidze S. et al. (2015) South African Guidelines Excellence (SAGE): Clinical practice guidelines - quality and credibility. Vol. 105.

28. Pre-Hospital Emergency Care Council. (2014) Clinical Practice Guidelines. Accessed 25th November 2016; Available from: http://www.phecit.ie/phecc/ clinical_resources/

clinical_practice_guidelines/2014_edition_cpgs/phecc/ exams_and_candidates/clinical_practice_guidelines/ clinical_practice_guidelines.aspx?hkey=23dd59a6-6b5b4d0b-9874-6e3467c026e 0.

29. Queensland Ambulance Service. (2016) Clinical Practice Guidelines. Accessed 25th November 2016; Available from: https://ambulance.qld.gov.au/CPGtable.html.

This is an Open Access article distributed under the terms of the Creative Commons Attribution-Non-Commercial-ShareAlike 4.0 International (http:// creativecommons.org/licenses/by-nc-sa/4.0/), which permits use, distribution, and reproduction in any medium, provided the original work and any attributes thereof are properly cited, are distributed under the same licence, and that the work is not used for commercial purposes. Content copyright remains with the authors, who grant the IJP a licence to reuse and distribute. 